\title{
High temperature fretting tribometer - study of the dynamic behaviour and tangential load measurement
}

\author{
J. Fortes Da Cruz ${ }^{1, a}$, I. Lemaire-Caron ${ }^{1}$, T. Da Silva Botelho ${ }^{1}$, A.-M. Durand ${ }^{2}$ \\ AND G. INGLEBERT ${ }^{1}$ \\ 1 LISMMA-SUPMECA, EA-2336, 3 rue Fernand Hainaut, 93400 Saint-Ouen, France \\ 2 ACM, 9 rue de la Gare, 78640 Villiers-Saint-Frederic, France
}

Received

\begin{abstract}
Experimental approaches to study fretting are heavily dependent on the performances of the test devices, especially considering the extensive use of quantitative parameters extracted from fretting loops. The dynamic response of a newly designed high temperature test bench is analysed to define the practical test range and identify dynamic disturbance sources. Displacement and load metrology are studied, leading to a direct comparison of two tangential load measurement methods. Accounting for the inertia loads and the elastic properties of the guiding system allows to increase the frequency operating range of the test bench.
\end{abstract}

Key words: Test rig analysis / fretting loops / metrology / high temperature

\section{Introduction}

The industrial frame for the design of this new high temperature fretting test device is aeronautical components subject to a vibratory environment. Aircraft components are severely strained because of the air flow around the fuselage and engine vibrations during normal flight. Take-off and landing phases also cause high amplitude shocks. These modern mechanical structures are submitted to ever harsher loading conditions, both static and dynamic, while also requiring longer lifespan, and lighter weight. These constraints activate different damage mechanisms on contacting surfaces submitted to relative motion such as bolted joints. Weight and static stiffness requirement are well satisfied using predictive numerical design methods, however, the dynamical behaviour is still largely unpredictable without building prototypes. This is due to the challenge of modelling the complex phenomena occurring at the contacting interfaces, which depend on many parameters: materials, machining process, normal load at the interface, working temperature; the interface response can change with the accumulation of damage. Fretting is a predominant source of damage in mechanical assemblies. It combines wear and fatigue at the contacting surface, and can lead to material loss [1] as well as crack nucleation and propagation [2].

\footnotetext{
a Corresponding author:

julien.fortesdacruz@supmeca.fr
}

Scientific fretting studies can be classified in three different categories [3]. Fundamental research tests (laboratory studies of elementary systems and geometries) are dedicated to understand the basic mechanisms of the tribological phenomena, and to develop and calibrate analytical and numerical models [4]. The second level is simulation tests, where contacting surfaces are kept elementary, but in which test conditions are chosen to be representative of real life operating conditions of a mechanical system. Finally we find validation tests for which part of or the whole mechanical system is rebuilt and tested. The two latter kinds of tests often yield application dependant results. Test rig dependence of the results is a major issue for dissemination and standardisation of test results among different laboratories. Their kinematic and dynamic behaviour and their influence on the contacting surfaces also need to be extensively understood in order to develop accurate theoretical models.

Since the end of the 90s, fretting damage assessment became more and more tied to energy based approach $[5,6]$, especially when wear mechanisms are involved. The tangential contact stiffness is also a key parameter for finite elements modelling of mechanical assemblies. Accurate fretting loops (tangential load versus relative displacement) are therefore required to obtain relevant results. Friction is generally supposed to be independent from the test frequency for metallic materials, so many studies focus on low frequency range test conditions 
(under $1 \mathrm{~Hz}$ or up to a few $\mathrm{Hz}$ ) to reduce the disturbances caused by the dynamic response of the test bench. Specifically designed test rigs $[7,8]$ can reach higher values (up to a few hundred $\mathrm{Hz}$ ). Li et al. [9] introduced the concept of deliberately reducing the stiffness of a part of the test bench to ensure a better metrology, while retaining high fundamental frequency of the mechanical assembly $(160 \mathrm{~Hz})$. These stiffness goals imposed the design of heavy parts, thus amplifying the inertia effect, which becomes significant above $40 \mathrm{~Hz}$. Matlik et al. [10] designed a high temperature fretting rig with bulk fatigue loading using piezo-electric stacks, and successfully reached operating tests frequencies above $600 \mathrm{~Hz}$.

Although it is widely accepted that rigidity and inertia of the test rigs have an effect on the accuracy of the results obtained, not many studies have been published about these issues. Each research laboratory has established and perfected its own set of design methods and know-how. This paper aims to go further in the understanding of the influence of the fretting test benches behaviour on tests results and the ensuing operating range limitations. We will present a way to expand the available frequency range by comparing two classical tangential load sensors position. The robustness of the load measurement method and effect of high temperature $\left(600{ }^{\circ} \mathrm{C}\right)$ will also be analysed.

\section{Test setup}

The new fretting tribometer (Vibro Thermo Tribometer - VTTM) is designed as an ambient and high temperature evolution of a cryotechnical environment fretting test rig (Vibro Cryo TriboMeter - VCTM) already in use at LISMMA laboratory [11]. It is based on the same principle, but optimized in order to increase the frequency, displacement amplitude and normal load operating range. The VCTM was designed for low amplitude $(\leq 20 \mu \mathrm{m})$ high contact pressure fretting fatigue tests, on ball on flat configurations. The VTTM is designed to study flat on flat contact configurations in gross sliding fretting conditions, focusing more on energy dissipation. The description of a few key technical solutions chosen for this bench will be accompanied by short reviews of the other kinds of solutions found in literature.

\subsection{General principle}

The tribometer is designed to apply two independent loadings: a static normal load and a cyclic tangential one. The normal load is applied via calibrated dead weights (from $10 \mathrm{~N}$ up to $400 \mathrm{~N}$ ) or a traction cable (from $150 \mathrm{~N}$ up to $1000 \mathrm{~N}$ ), and measured thanks to a strain gauge load cell. The tangential load is exerted by an electromagnetic actuator that can apply $500 \mathrm{~N}$ on a $1-5000 \mathrm{~Hz}$ frequency range and monitored by two piezoelectric load cells. Displacement amplitude with a span from $1 \mu \mathrm{m}$ up to $1 \mathrm{~mm}$ is measured via a specific high temperature contact-less
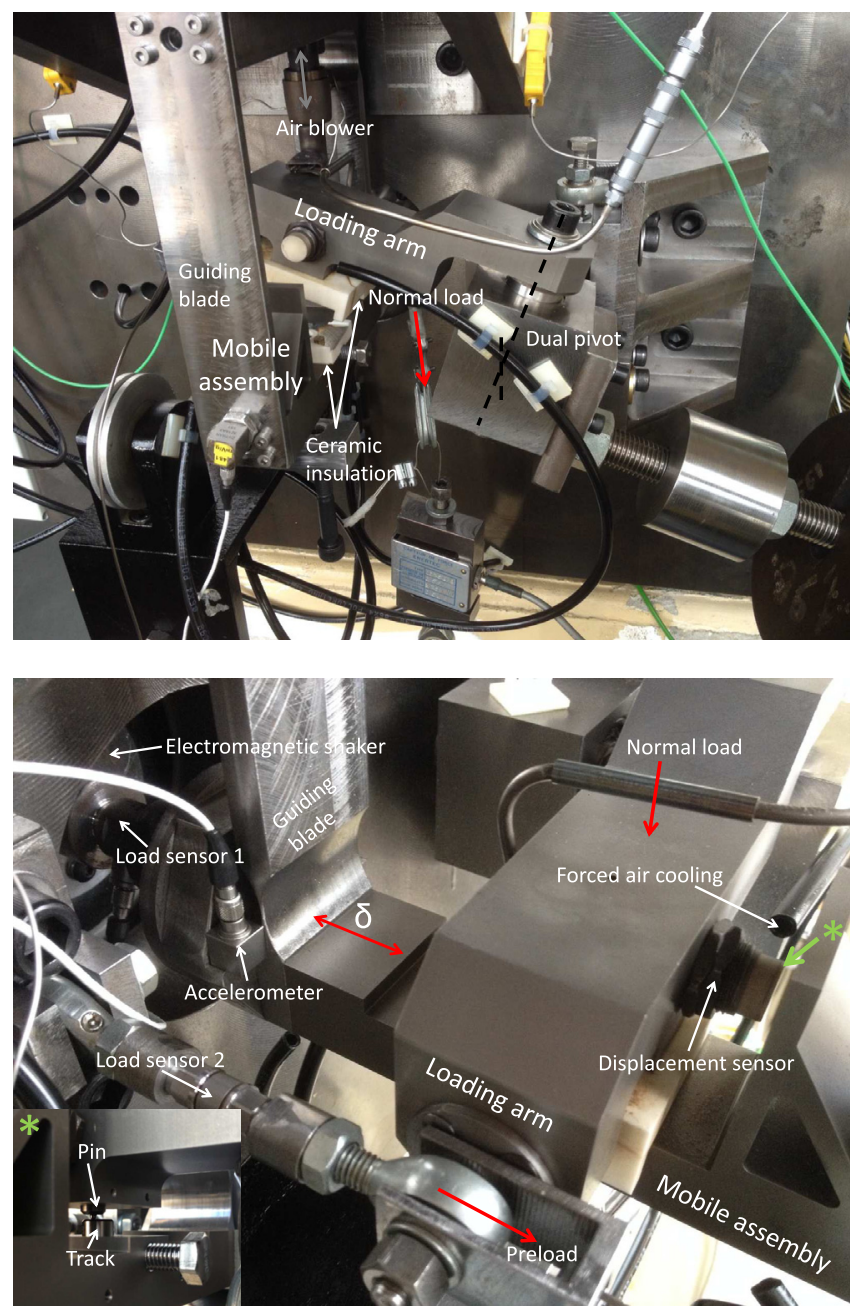

Fig. 1. Global test setup and sensors positions.

Eddy current sensor. Tests can be carried out in ambient temperature and up to $600{ }^{\circ} \mathrm{C}$ using a hot air blower with extra isolation parts around the specimen. Instead of cutting the heat flow by inserting isolation parts in the assembly, the mechanical parts exposed to high temperatures were machined from an Inconel 718 alloy in order to act as heat sinks in conjunction with forced air cooling.

\subsection{Displacement measurement}

Accurately measuring the relative displacement between the test specimen is one of the most important feature of a fretting tribometer. The range of relative displacements involved between the contacting surfaces can reach a few micrometers, and will rarely exceed a few hundred micrometers for most fretting industrial cases. Higher displacement amplitude would tend to be classified as reciprocal sliding study.

Different technologies can be found in the literature. They can be first separated between contact and contactless methods, the latter being preferable to limit the perturbations added by the extra contact point. Contact 


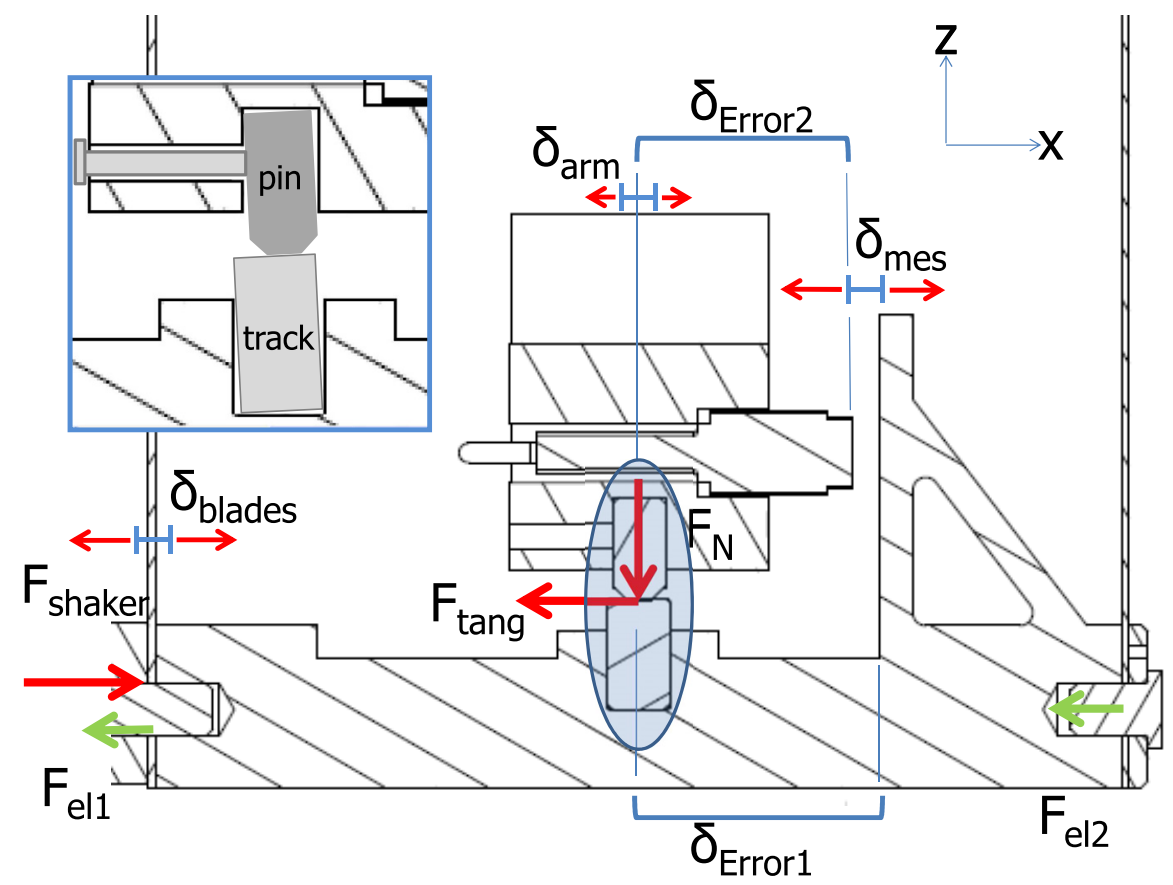

Fig. 2. Displacement metrology analysis.

measurement methods generally involve LVDT (Linear Variable Differential Transformer) sensors [12,13]. Contactless methods regroup Eddy current sensors [11] and optical methods: digital image correlation [12], LDV (Laser Doppler Vibrometers) [9, 14] and photonic optic fibres sensors [15]. Eddy current sensors require a specific target with size constraints, and compatible material requirements. LVD uses Doppler effect to measure target velocity, and photonic optic fibres sensors are based on the variation of the light intensity reflected by the surfaces which can be related to the distance to the transducer.

Another distinction can be made between direct and relative measurement methods. Kartal et al. [12] highlight that no matter how stiff the base and specimen holding assembly is designed, there will be some unwanted motion of the fixed specimen. The most accurate way to get the specimen motion is to use a relative measurement method. One way to proceed is to use two sensors [15], one aimed at the fixed specimen, the other at the moving one. A proper design can ensure that the mechanical chain between a unique sensor and its target is stiff enough to be considered as a rigid body.

The last key issue regarding displacement measurement is the proximity to the contact interface. The reference point and the target of the displacement sensor have to be as close as possible to the contacting surfaces of the specimen to reduce the disturbance of the test bench mechanical parts. Optical methods require heavy postprocessing, but they are most suited to satisfy this design constraint as long as a direct access to the specimen can be guaranteed. Specific environment application and the associated issues(insulation and heating system, immer- sion of the specimen) add a considerable technical and design challenge.

A detailed mechanical analysis of the measured displacement and repartition of the loads on our test bench is presented in Figure 2. If we set aside the inertia effects, the relations between the different forces can be expressed according to the following equation:

$$
F_{\text {shaker }}=F_{\text {el1 }}+F_{\text {el2 }}+F_{\text {tang }}
$$

The measured relative displacement $\delta_{\text {mes }}$ has two error components: $\delta_{\text {error } 1}$ between the surface of the flat target and the contacting surface of the track, and $\delta_{\text {error2 }}$ which is the displacement error between the active surface of the Eddy current sensor, and the contacting surface of the pin. The load repartition analysis indicates that no significant deformation of the loading arm can occur, therefore $\delta_{\text {error2 }}$ is mainly caused by pin bending and rotation as illustrated in the close up. However, $\delta_{\text {error } 1}$ is the combination of the track bending, its rotation and the elastic deformation of the track support under the action of $F_{\mathrm{el} 2}$. Depending on the test setup and conditions, this contribution can be noticeable, even though it remains a low influence parameter. The so-called "bench stiffness" is thus essentially due to bulk specimen related elastic behaviour, and their locking mechanism. Filippi [7] had come to a similar conclusion when he identified specimen rotation (parallax error) as a predominant source of experimental error. It depends on their shape and material, but also on the load applied by the locking screws. It would be advised to tighten the specimen with a torque wrench to improve the repeatability of the tests. 


\subsection{Tangential load measurement}

The other key parameter monitored for fretting tests is the tangential load transmitted through the surfaces in contact. For global sliding mode, this load gives access to the friction coefficient.

Fretting studies can be undertaken in a wide range of frequencies (rarely lower than $1 \mathrm{~Hz}$ up to a few hundred), but always involve dynamic phenomena. The typical load measurement method is a uni-directional piezoelectric load cell. 3D load cells can also be found in the literature [16]. Test-benches are often built around similar principles which are either dictated by practical design limitations, specific environment constraints, or based on previous scientific studies. Normalization process for fretting studies is still underway, and is more advanced for fretting-fatigue tests. For fretting-wear tests some essential design guidelines have already been established. As far as the tangential load is concerned, inertial loads resulting from the dynamic motion of the mechanical parts disturb the measurement of the actual tangential load at the contact interface. It is therefore advised to design the bench so that the measurement of the transmitted load occurs on the static specimen. The test bench presented in this study combines two piezo-electric load cells: one placed between the electromagnetic actuator and the moving mechanical assembly, and the other one between the static assembly and the base. Both sensors measure the tangential contact load. This dual setup enables a comparative study of the two different design choices and their limitations.

\section{Tangential force measurement methods comparison}

\subsection{Frequency range limitations in global slip regime}

An experimental determination of the dynamic response of the test rig is necessary to validate the global design $[9,17]$. It can also be used as a reference to assess the ageing of these components or to detect structural damage [18]. All the individual parts of the test bench were designed so that their first resonance mode is higher than the expected test frequency range. The lowest frequency, that of the base support, is estimated at about $300 \mathrm{~Hz}$. However no mechanical assembly can insure perfect bonds between the different parts so the behaviour of a full structure is more complex. The observation of the fretting loops obtained under different test conditions is an empirical way of checking the practical test range of the device. The accuracy of the loops can be evaluated based on the friction coefficient, the dissipated energy (area within the curve) and the tangential contact stiffness. The industrial frame required to compare the potential damping that different coatings and surface treatments provide, in fretting-wear and frettingfatigue conditions, without bulk fatigue loading. These tests were first started on an already existing frettingfatigue test bench, the VCTM and have been described in a conference paper [19].

Fretting loops in Figure 3 illustrate the observed disturbance (thin blue line) of the tangential load signal, which appeared for global sliding regime only. Frequency sensitivity tests have been carried out: the higher the frequency, the more intense the disturbance grows. At $80 \mathrm{~Hz}$, the amplitude of the disturbance is even greater than the friction force (Fig. 3d). Lowering the test frequency at $20 \mathrm{~Hz}$ revealed a dampened behaviour (Fig. 3a). The frequency of the sinusoidal signal was estimated at about $400 \mathrm{~Hz}$, and remains constant even when the test frequency varies. The new test bench presented in this article was designed in order to reduce this disturbance. The results obtained on the VTTM in similar test conditions (red dashed lines) show a noticeable improvement. However, the phenomenon is still present at frequencies higher than $35 \mathrm{~Hz}$, and the disturbance shows a more complex behaviour with multiple harmonics. Our analysis of the synchronized load, displacement and acceleration signals showed that the disturbance is initiated at the beginning of the reverse motion. Multiple characterization tests were carried out in order to identify potential sources: the influence of the guiding blades stiffness, the mass of the mobile assembly, the loading arm's dual pivot bearings tightening, the base support bracket. Ramalho et al. [17] had identified a resonance mode of the loading arm which caused a similar phenomenon. Our test rig analysis seems to point towards incriminating the first mode of the base support as the main cause of disturbance. Even massive base supports that could be considered as rigid bodies can influence the bench behaviour which is another design constraint that has to be considered. From these observations, we can establish a conservative empirical design rule for fretting wear tests: the first resonance mode of each individual part (save for the guiding system) should be at least 10 times the desired maximum test frequency in global sliding regime.

\subsection{Tangential load measurement}

\subsubsection{Mechanical analysis}

Figure 4 displays a schematic view of the test setup and the forces involved. The dual sensor setup allows a direct comparison of two tangential load $F_{\mathrm{t}}$ measurement methods. We calculate $F_{\mathrm{t} 1}$ using sensor $(1)$ (signal $F_{\text {mes1 }}$ ) which is inserted between the oscillating part holding the track and the electromagnetic actuator. $F_{\mathrm{t} 2}$ results from sensor $(2)$ (signal $F_{\text {mes1 }}$ ) which is fixed to the base and holding the pin-holding arm in place with a preloaded ball joint according to the most common design.

The reference method uses load sensor 2 signal to track the transmitted load through the contact. The position of the sensor ensures a direct measurement of the transmitted load.

$$
F_{\mathrm{t} 2}=F_{\mathrm{mes} 2} \frac{L 1+L 2}{L 2}+F_{i 2} \approx \frac{F_{\mathrm{mes} 2}}{0.64}
$$




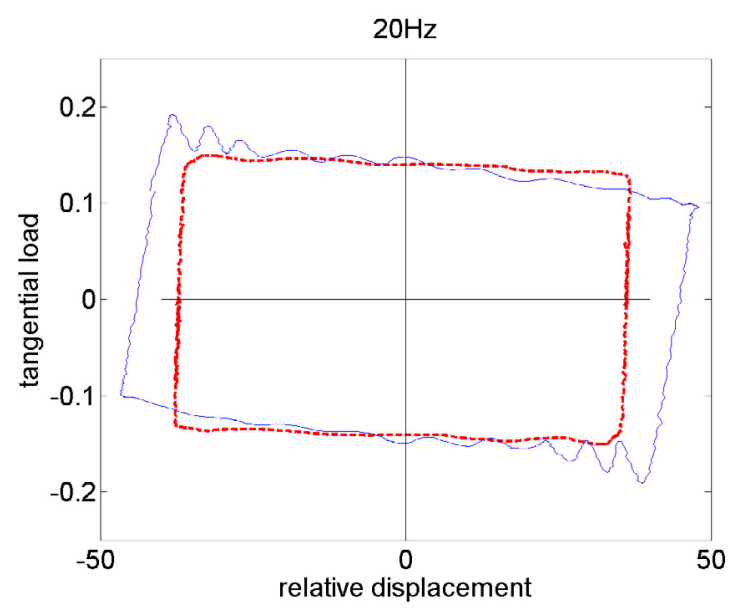

(a)

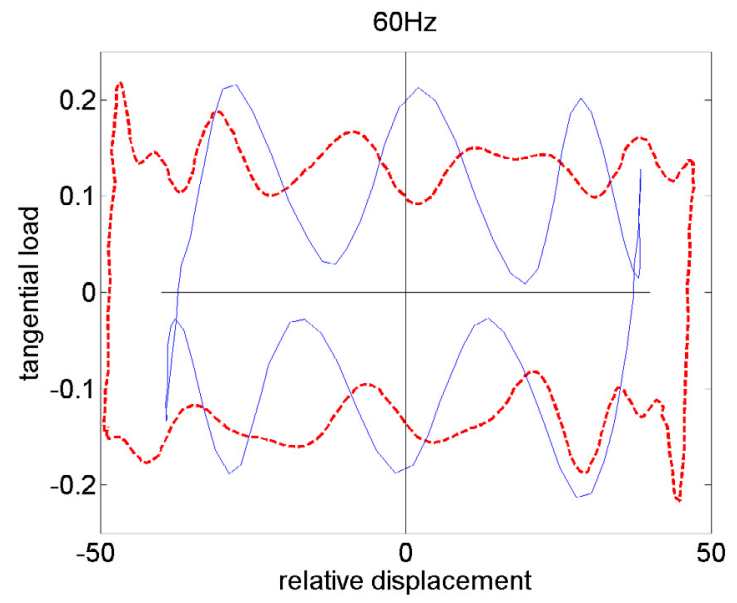

(c)

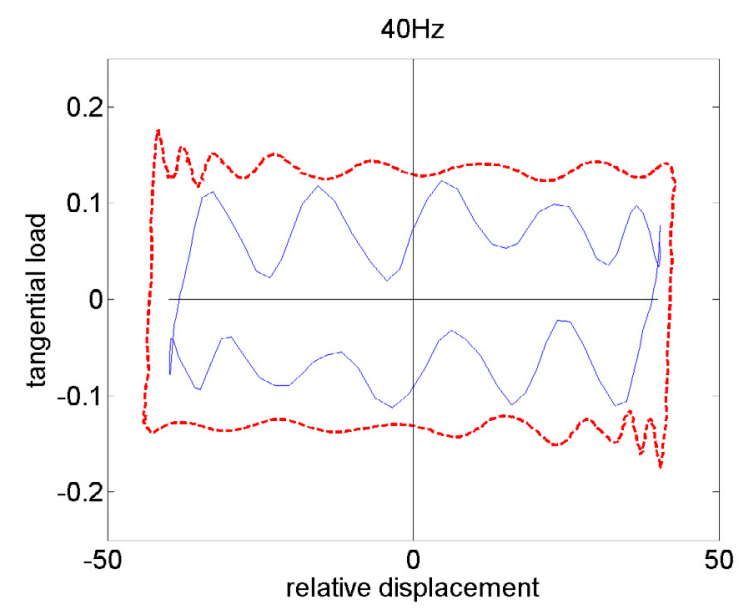

(b)

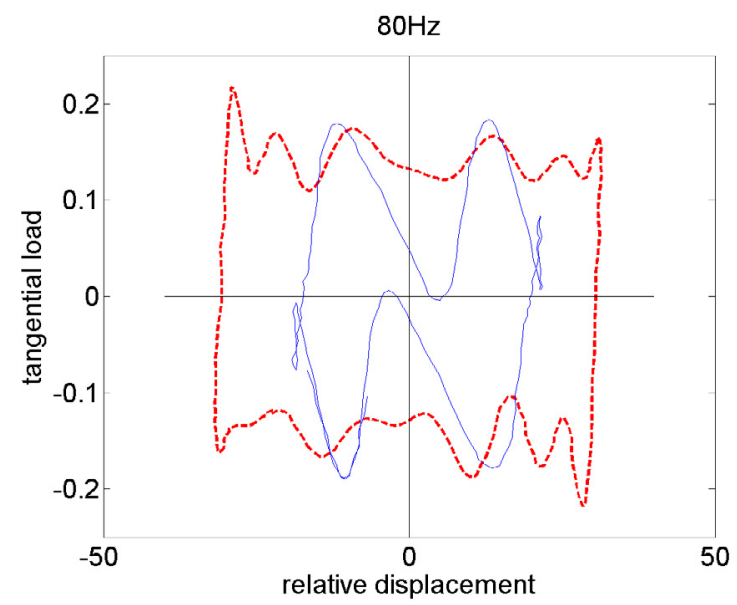

(d)

Fig. 3. Global sliding fretting loops on the VCTM (blue - solid lines) and VTTM (red - dashed lines) test benches at 20, 40, 60 and $80 \mathrm{~Hz}$.

The acceleration levels measured close to the loading arm's (7) center of gravity lead us to neglect these inertia effects for this study. The dual pivot guiding mechanism also opposes a negligible resistance compared to the measured tangential contact forces. The design of the different parts ensured that no significant disturbing load pollutes the signal (resulting torque or extra friction forces).

The new method proposed measures the load exerted by the actuator $F_{\text {shaker }}$. The signal acquired by the sensor $F_{\text {mes } 1}$ is not equal to the tangential load $F_{\mathrm{t} 1}$ at the contact interface but also includes the elastic reaction $F_{\text {el1 }}$ and $F_{\mathrm{el} 2}$ of the guiding blades (6) and the inertial forces of the moving assembly (8) $F_{i}=-\gamma m_{\text {mobile. Instead of lim- }}$ iting the operating range so that they can be neglected, we implemented a correction protocol accunting for these contributions. Writing the fundamental principle of the dynamics on the system $\{$ track + support $\}$ results in the following equations:

$$
F_{\mathrm{t} 1}=F_{\mathrm{mes} 1}-F_{\mathrm{el} 1}-F_{\mathrm{el} 2}-\gamma m_{\text {mobile }}
$$

with the following expressions for the elastic forces:

$$
F_{\text {el }_{\mathrm{i}}}=K_{\text {blades }_{\mathrm{i}}}(f, N) \delta_{\text {blades }_{\mathrm{i}}}
$$

The stiffness of the blade guided oscillating assembly might depend on the test frequency $f$ and the applied normal load $N$. A contact-less frequency characterisation of the behaviour of this assembly was done for two different blades setups. The results are detailed in the next paragraph. The displacement $\delta$ of the mobile assembly is not exactly equivalent to the relative displacement $\delta_{r}$ measured by the test setup (Fig. 2). The loading arm was designed so as to have a rigid body behaviour in the expected range of test conditions. However it might undergo some elastic deformations $\delta_{\text {arm }}$ under the tangential loading transmitted through the contact. The real blades displacement is expressed as $\delta_{\text {blades }}=\delta_{r}+\delta_{\text {arm }}$. Tests under severe conditions were carried out and resulted in negligible $\delta_{\text {arm }}$ values. We will not add this correction term for the rest of this study. The elastic contributions of the two blades will also be regrouped. The acceleration is measured thanks to a piezoelectric accelerometer 


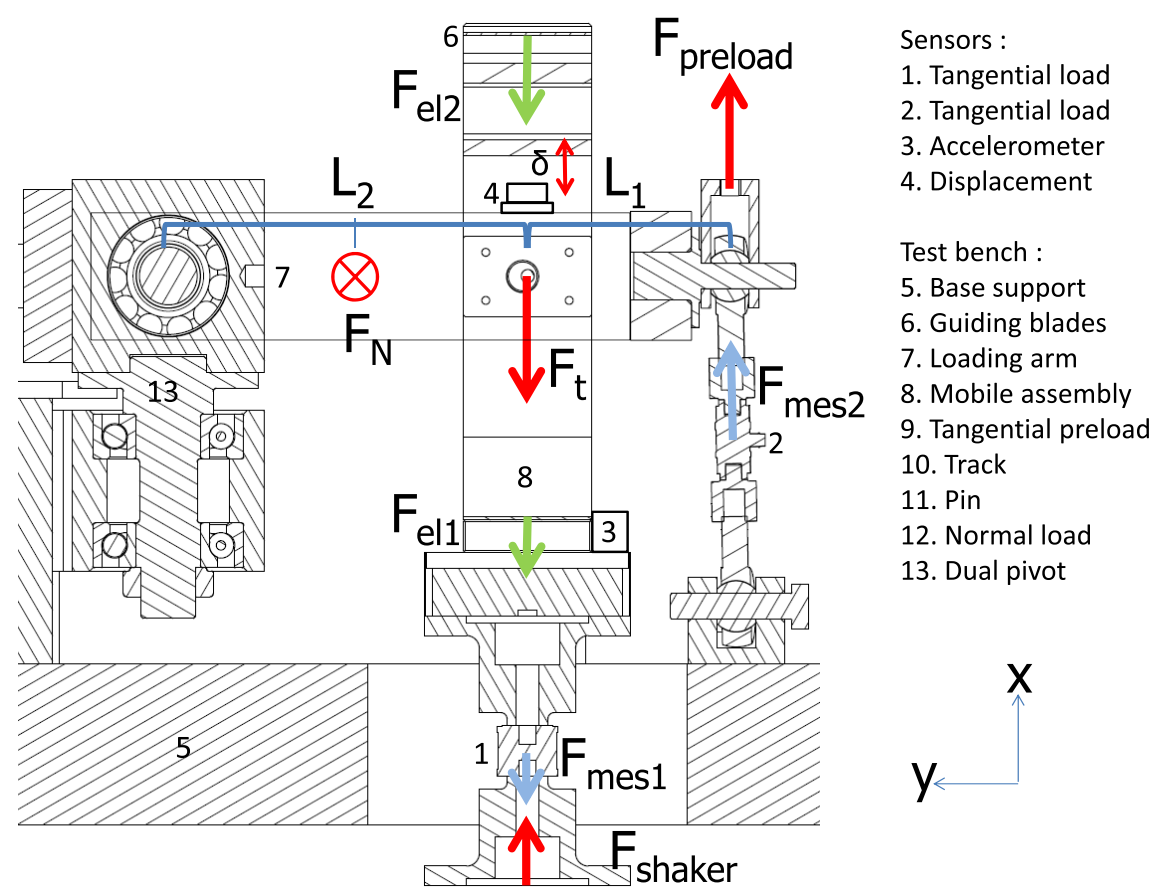

Fig. 4. Mechanical analysis of the test setup (in the contact plane).

positioned as shown in Figure 4. Other accelerometers were positioned in different places during the validation process of the bench; they showed no relevant cross direction acceleration. The mass $m_{\text {mobile }}$ of the moving part can be estimated from the electromagnetic actuator data sheets and the weighing of the different components of the assembly. It was estimated at $2.67 \mathrm{~kg}$ with less than $5 \%$ error. However, the dynamic contribution of the mass may be different from its static value.

\subsubsection{Mobile assembly characterization}

In order to correct the $F_{\text {mes1 }}$ signal, $K_{\text {blades }}$ and $m_{\text {mobile have to be determined. The mobile assembly is a }}$ mass spring system, with theoretically no damping when the contact is unloaded. However, it is composed of multiple parts assembled together, causing some energy dissipation at the junctions on top of the internal damping of the bulk materials. It is known that structural damping of bulk metallic materials is around $0.01 \%$ so we will neglect these damping sources at first. The previous equation can be written this way:

$$
F_{\text {mes1 }}=F_{\text {el }}+\gamma m_{\text {mobile }}=K_{\text {blades }} \delta+m_{\text {mobile }} \ddot{\delta}
$$

Performing a Fourier transform gives:

$$
\frac{\hat{\delta}}{F_{\text {mes } 1}^{\hat{n}}}=\frac{1}{K_{\text {blades }}-\omega^{2} m_{\text {mobile }}}
$$

with $\omega_{0}=\sqrt{K_{\text {blades }} / m_{\text {mobile }}}$ the fundamental resonance frequency.
Table 1. Identified $K_{\text {blades }}$ and $m_{\text {mobile }}$ resulting from the FFT analysis.

\begin{tabular}{ccc}
\hline Test setup & Rigid blade & Compliant blade \\
\hline$K_{\text {blades }}$ FFT $(\mathrm{N} . \mathrm{mm})$ & 398 & 21 \\
$m_{\text {mobile }}(\mathrm{kg})$ & 2.17 & 2.23 \\
\hline
\end{tabular}

Two blade setups (a stiff one and a compliant one) were designed according to Henein's work [20] and tested. Figure 5 presents the FFT (Fast Fourrier Transformation) of the signal for the two setups at ambient temperature (blue continuous line) and high temperature $\left(600^{\circ} \mathrm{C}\right)$ for the rigid blade (green continuous line). The FFT of a theoretical perfect mass spring system is also plotted, using the $K_{\text {blades }}$ and $m_{\text {mobile }}$ identified on the experimental curves.

The blade stiffness and mobile mass values are reported in Table 1.

The close up on the resonance peak shows that the system has a very limited damping. For the prismatic blades, the results are less accurate because of the lower input voltage, and lower amplitude of the measured tangential force. The inertial mass contributions are inferior to the static mass of the mobile assembly. The design of the bench ensures that even high temperature tests do not significantly alter the behaviour of the mobile assembly.

\section{Experimental validation}

\subsection{Materials and specimens}

The following experimental fretting loops were extracted from various test campaigns. Ball-on-plane and 


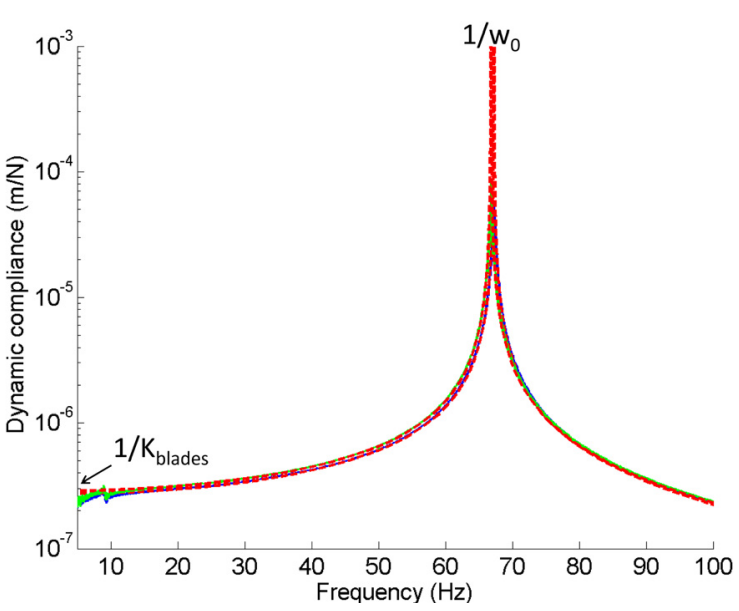

(a)

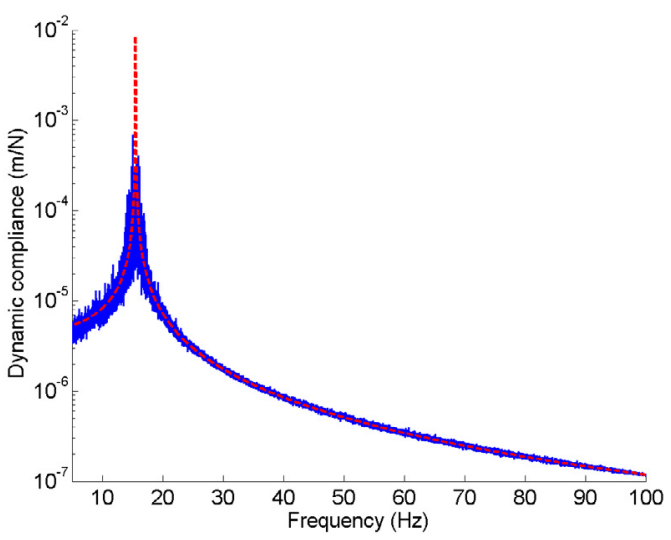

(c)

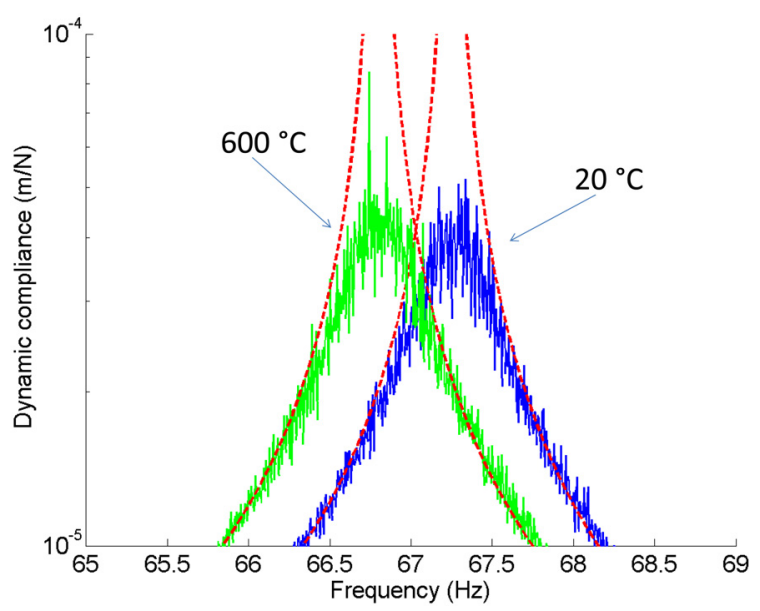

(b)

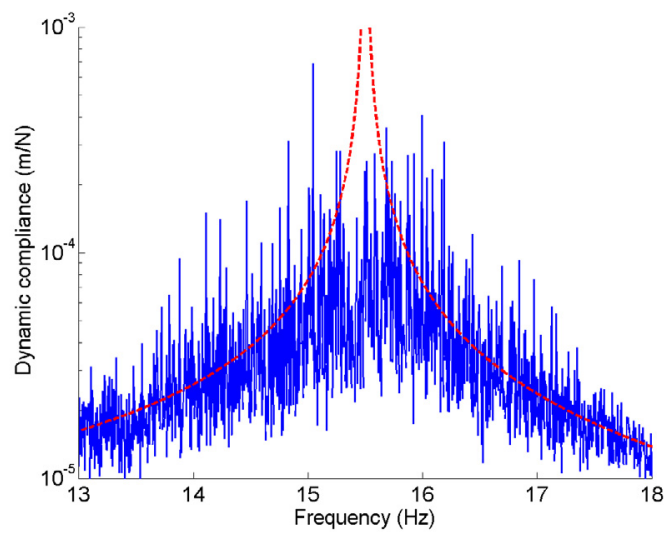

(d)

Fig. 5. FFT of the tangential load for the 1-100 Hz sweep, and theoretical FFT (dashed red line) plotted with the identified parameters $m_{\text {mobile }}$ and $K_{\text {blades }}$. (a) Rigid blade. (b) Zoom in. (c) Compliant blade. (d) Zoom in.

plane-on-plane contact configurations involved different materials (Inconel 718, Ti6Al4V, stainless steel), surface treatments (DLCs, WS2 ionic implantation) and test conditions (normal load, frequency, amplitude and temperature).

\subsection{Fretting loops analysis}

Fretting loops are the reference tool for qualitative fretting studies. The loops using $F_{\mathrm{t} 1}, F_{\mathrm{t} 2}$ and $F_{\text {mes } 1}$ were plotted in Figure 6 for global sliding test conditions corresponding to those in Figure 3. The contribution of the inertial forces was also singled out for $F_{\mathrm{t} 1}$.

A key result from these tests is the shape of the $F_{\mathrm{t} 1}$ fretting loops. $F_{\mathrm{t} 2}$ reference loops were noticeably disturbed by the vibration issues identified earlier at frequencies superior to $35 \mathrm{~Hz}$. The sensor measuring $F_{\text {mes1 }}$ is aligned on a vibration node of the base structure resulting in cleaner loops. With a simple and robust correction methodology, using this signal increases the available range of frequency for global sliding fretting tests to at least $80 \mathrm{~Hz}$. Further testing would be required for a more precise characterization of the practical frequency range.

\subsection{Robustness of the correction protocol}

\subsubsection{Blades stiffness}

The dynamic behaviour of the mobile assembly may be modified when the contacting surface of the specimen is loaded. In order to study its sensitivity to frequency, normal load and specimen configuration, another stiffness identification protocol was tested. Figure 6 a illustrates the measurement of $K_{2}$ blade stiffness value, using the stable sliding phase as a reference. This part of the fretting cycle is supposed to be horizontal provided surface damage is limited. $K_{2}$ is calculated by a mean square regression. These tests aim to study the influence of the frequency and the normal load on the behaviour of the mobile assembly. A stable global sliding fretting regime should be 


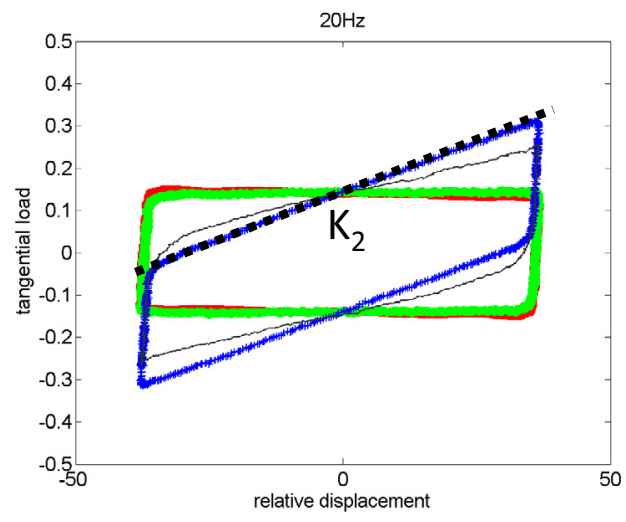

(a)

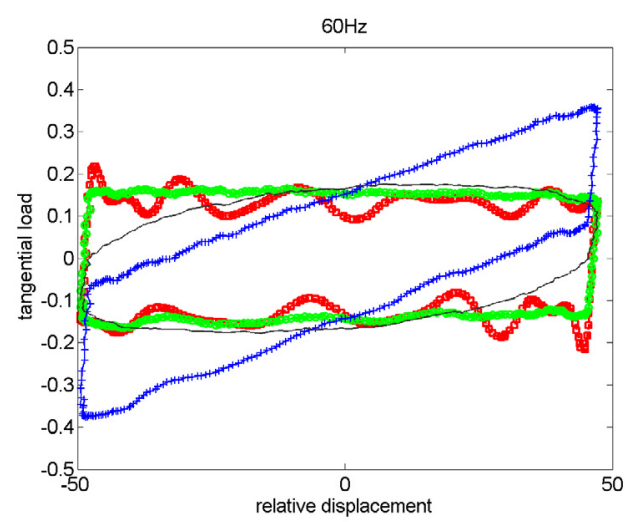

(c)

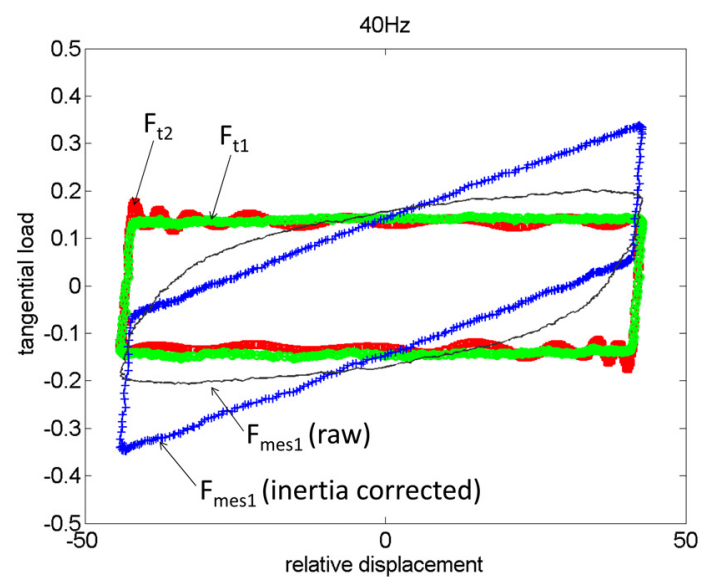

(b)

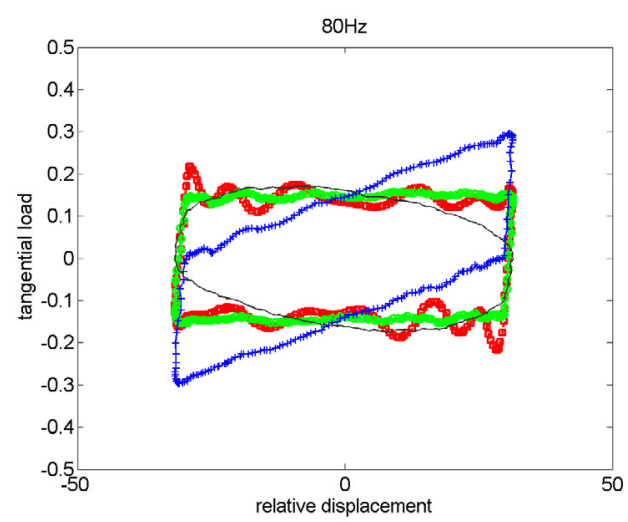

(d)

Fig. 6. Global sliding fretting loops using $F_{\mathrm{t} 2}$ (red squares), $F_{\mathrm{mes} 1}$ (grey thin line), inertia corrected $F_{\text {mes } 1}$ (blue crosses) or $F_{\mathrm{t} 1}$ (green circles).

achieved for these tests in order to measure the $K_{2}$ stiffness introduced earlier. Wear has to be limited, and the sliding phase as flat as possible. Since uncoated contacts are tested, we can expect a severe damaging tribological behaviour. Correct conditions are achieved by applying a low normal load $(10 \mathrm{~N})$, and adding a drop of lubricant at the contact interface to improve friction. As a consequence, the friction force generated by these tests conditions was low, approaching the lower range of the bench capabilities. We repeated the process using uncoated titanium on steel specimen in a plane-on-plane contact configuration, with a normal load increased to $150 \mathrm{~N}$ in order to obtain higher friction force values. The $K_{2}$ values for previous sliding tests ( $80 \mathrm{~N}$ load) were also calculated. All results obtained are displayed in Table 2.

Standard deviation of the measured values is lower than $5 \mathrm{~N} . \mathrm{mm}^{-1}$. We could not achieve a global sliding regime at $80 \mathrm{~Hz}$ for the $150 \mathrm{~N}$ load because of the cumulative damage of the specimen. Even with the drop of lubricant, the high normal load caused severe wear of the contacting surfaces. The frequency and the normal load's influence on the elastic behaviour of the mobile assembly is low in the range of our test conditions within a $5 \%$ er-
Table 2. Measured blades stiffness versus test frequency.

\begin{tabular}{cccccc}
\hline $\begin{array}{c}\text { Frequency } \\
(\mathrm{Hz}) / \text { load }\end{array}$ & Contact type & 20 & 40 & 60 & 80 \\
\hline$K_{\text {blades }}(\mathrm{N} . \mathrm{mm})$ & unloaded & \multicolumn{5}{c}{398} \\
$K_{2-10 \mathrm{~N}}(\mathrm{~N} . \mathrm{mm})$ & ball/flat & 384 & 385 & 381 & 404 \\
$K_{2-80 \mathrm{~N}}(\mathrm{~N} . \mathrm{mm})$ & ball/flat & 408 & 407 & 404 & 404 \\
$K_{2-150 \mathrm{~N}}(\mathrm{~N} . \mathrm{mm})$ & flat/flat & 382 & 385 & 388 & - \\
\hline
\end{tabular}

ror margin. If we consider the range of the stiffness values expected for the studied materials $\left(10^{4} \mathrm{~N} . \mathrm{mm}\right)$, and the parallel contribution of this term, we can consider these variations negligible.

\subsubsection{Sensitivity to the identified parameters}

We ran a sensitivity study of the shape of the fretting loops to $K_{\text {blades }}$ and $m_{\text {mobile }}$ values. The dissipated energy, the friction coefficient and the tangential stiffness values were chosen as a quantitative reference. Figure 7 illustrates the resulting loops for $F_{\mathrm{t} 1}$ (green) and $F_{\mathrm{t} 2}$ (red) 


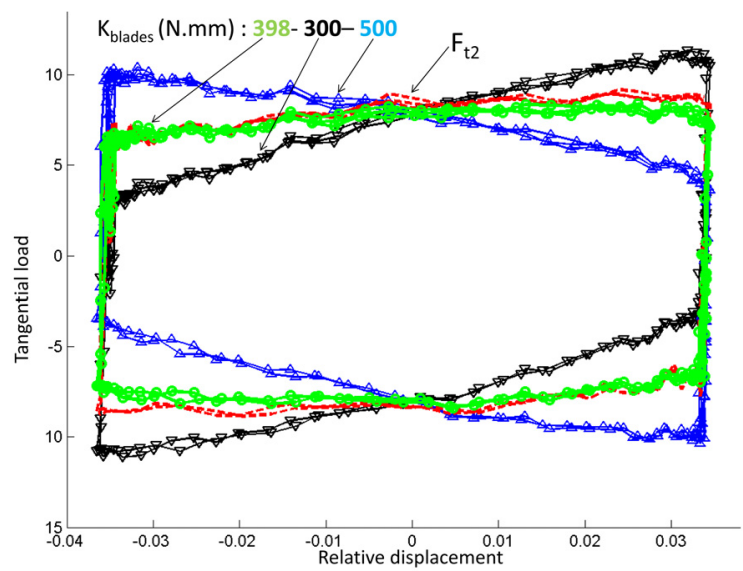

(a)

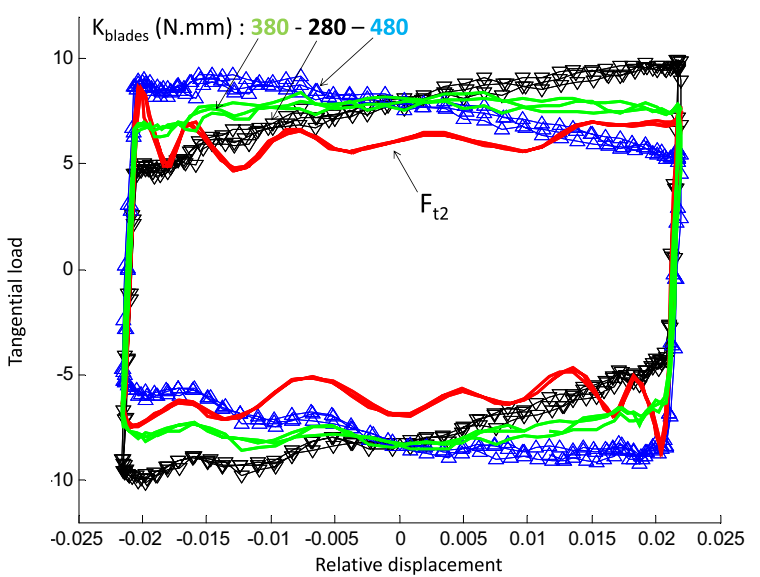

(c)

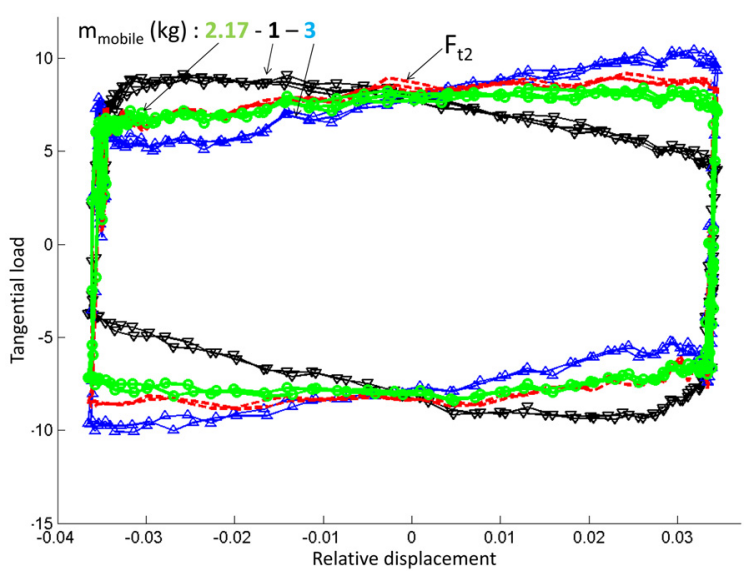

(b)

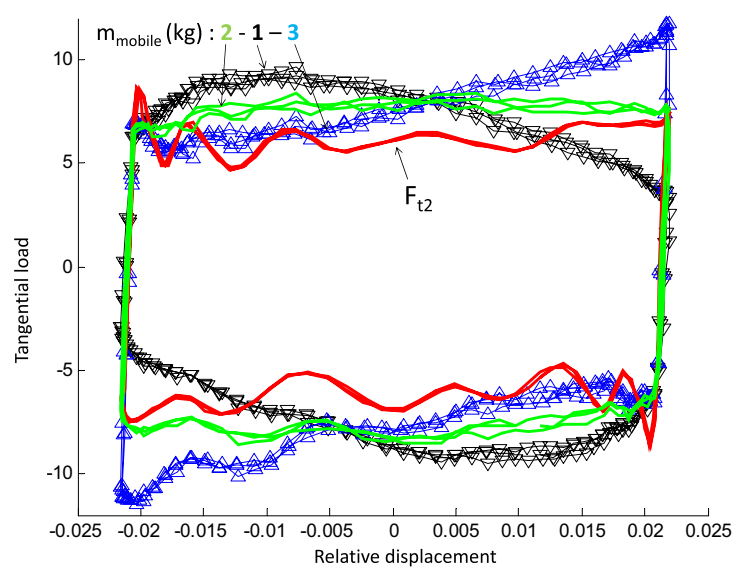

(d)

Fig. 7. Influence of the identified correction parameters value on the shape of the fretting loops in global sliding regime at 30 and $50 \mathrm{~Hz}$. (a) $K_{\text {blades }}$ influence $-30 \mathrm{~Hz}$. (b) $m_{\text {mobile }}$ influence $-30 \mathrm{~Hz}$. (c) $K_{\text {blades }}$ influence $-50 \mathrm{~Hz}$. (d) $m_{\text {mobile }}$ influence $50 \mathrm{~Hz}$.

at 30 and $50 \mathrm{~Hz} . F_{\mathrm{t} 1}$ signal was also plotted for a $100 \%$ variation of either $K_{\text {blades }}$ or $m_{\text {mobile }}$.

$K_{\text {blades }}$ variations do not affect the inside area of the loops (dissipated energy $E_{\mathrm{d}}$ ) or the mean value of the friction coefficient. It may however impact the standard deviation of the latest. $m_{\text {mobile variations do not signif- }}$ icantly alter $E_{\mathrm{d}}$ value either: a $100 \%$ uncertainty only causes under $2 \%$ spread. Uncertainty on both parameters do not affect the tangential stiffness calculated on the elastic unloading phase of the loops. At $30 \mathrm{~Hz}$ the difference between $E_{\mathrm{d} 1}\left(F_{\mathrm{t} 1}\right.$ loop$)$ and $E_{\mathrm{d} 2}\left(F_{\mathrm{t} 2}\right.$ loop$)$ is less than $5 \%$. However at $50 \mathrm{~Hz}$, it can reach up to $20 \%$.

\subsubsection{Test conditions}

The correction method was applied on the $F_{\text {mes1 }}$ signal acquired on previous test campaigns covering a wide range of test conditions. Figure 8 compares the fretting loops obtained for both measurement methods.

At low frequency $(10 \mathrm{~Hz})$ and high displacement amplitude $(500 \mu \mathrm{m})$ the guiding blades stiffness is the main contribution (Fig. 8a), and both loops are nearly identical. As the frequency increases $(30 \mathrm{~Hz})$ but remains in the acceptable operating range for the reference signal $\left(F_{\mathrm{t} 2}\right)$, the new measurement method improves noticeably the shape of the curve, eliminating small amplitude oscillations during the sliding phase (Fig. 8b). Above the frequency limit $(50 \mathrm{~Hz})$, the dynamic disturbance is negligible in partial slip regime (Fig. 8c). However in global sliding regime (Fig. 8d), we find the expected heavy distortion of the $F_{\mathrm{t} 2}$ loops, which is reduced on the corrected $F_{\mathrm{t} 1}$ loop. We can identify a different behaviour depending on the direction of the motion.

\section{Conclusion}

This work focused on a better understanding of the influence of the test bench behaviour on fretting tests results.

- The dynamic response of test rigs is a key issue for fretting studies, which involve very small amplitude 


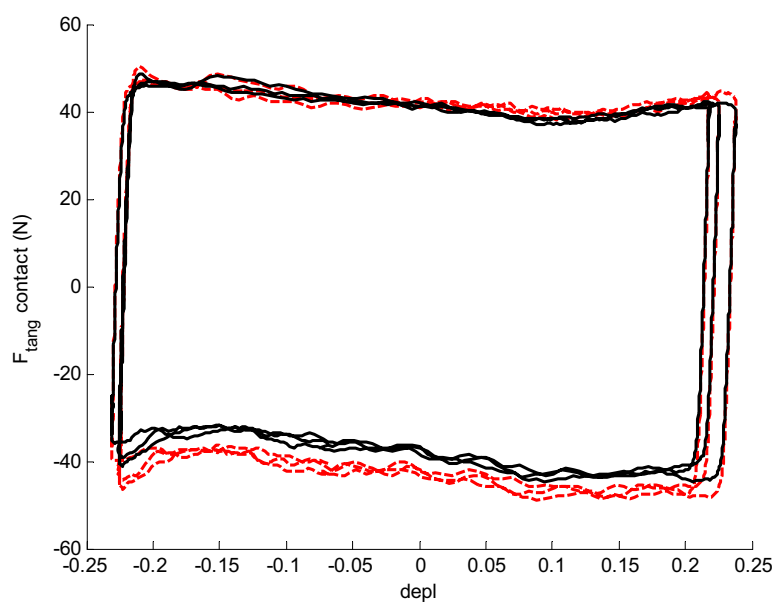

(a)

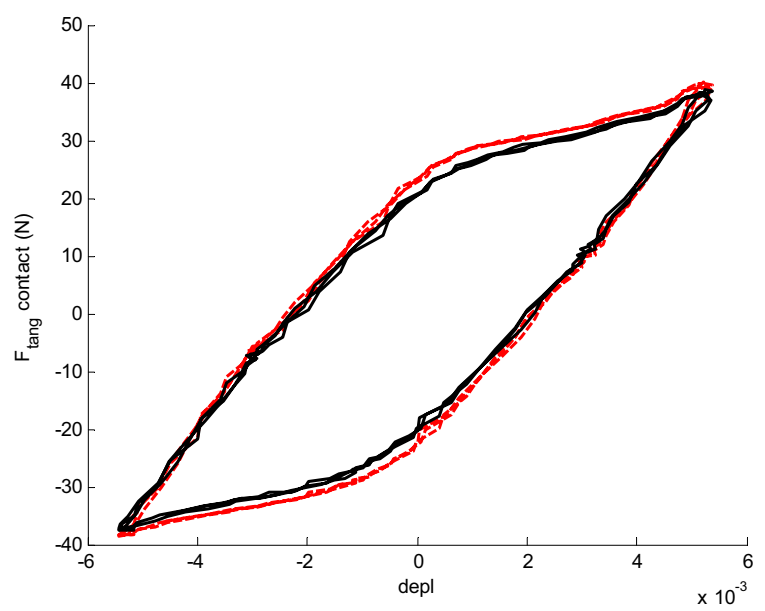

(c)

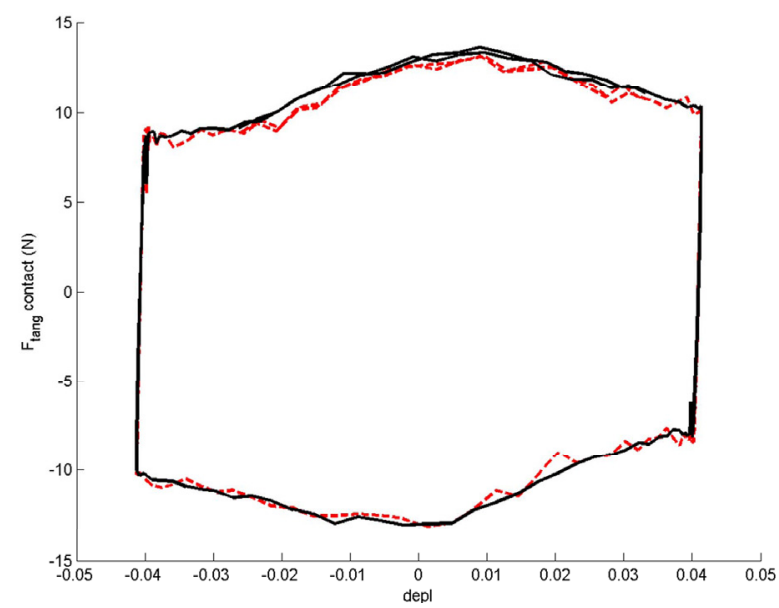

(b)

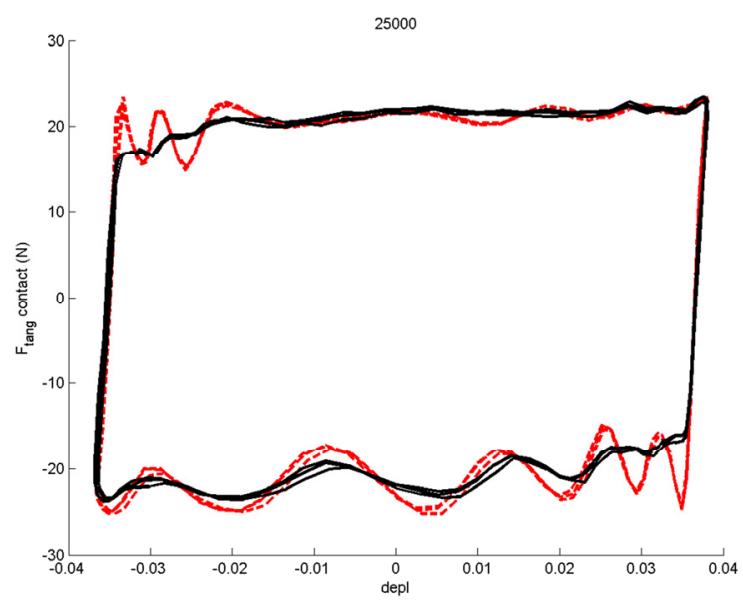

(d)

Fig. 8. Comparisom of fretting loops for $F_{\mathrm{t} 2}$ (red dashed lines) or $F_{\mathrm{t} 1}$ load (black lines) for different test cases (frequency, displacement amplitude, normal load, materials, fretting regime). (a) $10 \mathrm{~Hz}-20{ }^{\circ} \mathrm{C}-5 \mathrm{MPa}$. (b) $30 \mathrm{H}-20{ }^{\circ} \mathrm{C}-350 \mathrm{MPa}$. (c) $50 \mathrm{~Hz}-600{ }^{\circ} \mathrm{C}-5 \mathrm{MPa}$. (d) $50 \mathrm{~Hz}-600{ }^{\circ} \mathrm{C}-5 \mathrm{MPa}$.

alternative motion. The different parts as well as the global assembly have to be designed in order to rise the resonance frequencies at least an order of magnitude above the expected operating range. Depending on the design choices, even massive base supports can cause unwanted disturbance.

- To be able to extract accurate and inherent material response results, the usual design approach limits the operating range so that the different influence parameters are negligible compared to the contact behaviour. Unfortunately, financial limitations or technical challenge can make it hard to reach high test frequencies (very useful for fatigue analysis) for global sliding regime studies, especially when high normal loads are expected.

- A dual load sensor setup enabled a direct comparison between two positions: on the fixed arm (reference position) or on the mobile assembly. For the latter, iner- tial forces and elastic behaviour of the guiding system have been taken into account.

- A simple and robust unloaded characterization protocol for the mass-spring behaviour of the mobile assembly was implemented. The fretting loops plotted using this measurement method remain accurate for higher operating frequencies (up to at least $80 \mathrm{~Hz}$ when the standard setup limit is $30 \mathrm{~Hz}$ ) and lower normal loads, greatly extending the capabilities of the device.

- The newly designed test rig with the proposed load measurement method allows fretting fatigue tests (in sticking or partial slip regime) up to a few hundred $\mathrm{Hz}$, and fretting-wear type tests up to at least $100 \mathrm{~Hz}$. The upper frequency limit is still being studied.

These results show that different design approaches may be undertaken for fretting test benches. The loading arm usually combines a normal loading and tangential load measurement functions. Eliminating the latter function for this part would eliminate the need for one degree of 
freedom and help achieve wider operating range. Combined with normalized test rigs characterization protocols, it would be a step to deliver more absolute contact behaviour results, and facilitate comparative studies between different laboratories and test equipments.

Acknowledgements. The authors would like to thank the Pole ASTech Paris Region (FUI9) and MAIAS project (Maitrise des AmortIssements dans les ASsemblages) partners who supported these research.

\section{References}

[1] R.B. Waterhouse, Fretting wear, Wear 100 (1984) 107118

[2] R.B. Waterhouse, Fretting fatigue, Elsevier Science \& Technology, 1981

[3] Y.-M. Chen, C. Richard, Tribometres et essais tribologiques, Techniques de l'ingenieur, base documentaire: TIB466DUO. (2011). Fre

[4] R.W. Neu, Progress in standardization of fretting fatigue terminology and testing, Tribol. Int. 44 (2011) 1371-1377

[5] M.J. Pettigrew, M. Yetisir, N.J. Fisher, B.A. Smith, C.E. Taylor, Prediction of vibration and fretting-wear damage: an energy approach, ASME-Publications-PVP 389 (1999) 283-290

[6] S. Fouvry, C. Paulin, T. Liskiewicz, Application of an energy wear approach to quantify fretting contact durability: Introduction of a wear energy capacity concept, Tribol. Int. 40 (2007) 1428-1440

[7] S. Filippi, M.M. Gola, A. Akay, Measurement of tangential contact hysteresis during microslip, J. Tribol. 126 (2004) 482-489

[8] V. Chaudhry, S.V. Kailas, Damage quantification under sliding and seizure condition using first-of-a-kind fretting machine, Wear (2013)

[9] L. Li, I. Etsion, F.E Talke, The effect of frequency on fretting in a micro-spherical contact, Wear 270 (2011) $857-865$
[10] J.F. Matlik, T.N. Farris, F.K. Haake, G.R. Swanson, G.C. Duke, High-frequency, high-temperature frettingfatigue experiments, Wear 261 (2006) 1367-1382

[11] A. Koenen, P.H. Virmoux, R. Gras, J. Blouet, J.M. Dewulf, J.M. Demonicault, A machine for fretting fatigue and fretting wear testing in cryotechnical and normal environment, Wear 197 (1996) 192-196

[12] M.E. Kartal, D.M. Mulvihill, D. Nowell, D.A. Hills, Measurements of pressure and area dependent tangential contact stiffness between rough surfaces using digital image correlation, Tribol. Int. 44 (2011) 1188-1198

[13] A.M. Korsunsky, K. Kim, Dissipated energy and friction coefficient evolution during fretting wear of solid lubricant coatings, Tribol. Int. 43 (2010) 861-867

[14] C.H. Hager Jr, J.H. Sanders, S. Sharma, Effect of high temperature on the characterization of fretting wear regimes at ti6al4v interfaces, Wear 260 (2006) 493-508

[15] Y. Yoon, I. Etsion, F.E. Talke, The evolution of fretting wear in a micro-spherical contact, Wear 270 (2011) 567575

[16] M. Eriten, C.-H. Lee, A.A. Polycarpou, Measurements of tangential stiffness and damping of mechanical joints: Direct versus indirect contact resonance methods, Tribol. Int. 50 (2012) 35-44

[17] A. Ramalho, J.-P. Celis, Fretting laboratory tests: Analysis of the mechanical response of test rigs, Tribol. Lett. 14 (2003) 187-196

[18] A.S. Adamou, Comportement tribologique et réactivité de l'alliage 718 en atmosphere contrôlée et à haute température, $\mathrm{Ph}$.D. thesis, 2005

[19] J. Fortes Da Cruz, I. Lemaire-Caron, G. Inglebert, A.M. Durand, R. Merhej, Combined study of damage and damping phenomenon in coated contacts at ambient temperature, In: ASME 2012 11th Biennial Conference on Engineering Systems Design and Analysis, American Society of Mechanical Engineers, 2012, pp. 599-603

[20] S. Henein, Conception des structures articulées à guidages flexibles de haute precision, Ph.D. thesis, STI, Lausanne, 2000 